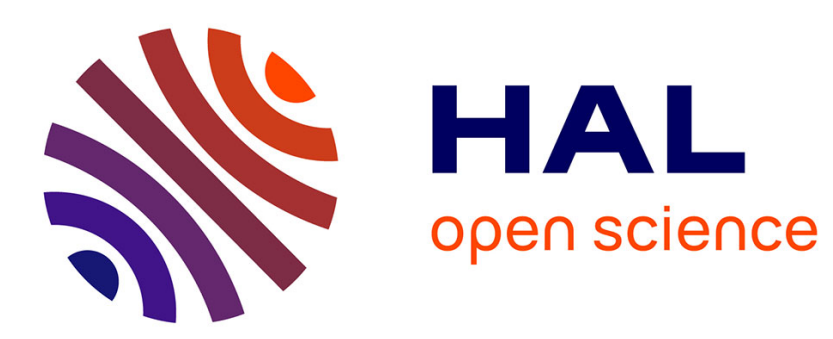

\title{
Taille des entreprises et salaires
}

Gilles Margirier

\section{- To cite this version:}

Gilles Margirier. Taille des entreprises et salaires. 2005. halshs-00094906

\section{HAL Id: halshs-00094906 https://shs.hal.science/halshs-00094906}

Submitted on 15 Sep 2006

HAL is a multi-disciplinary open access archive for the deposit and dissemination of scientific research documents, whether they are published or not. The documents may come from teaching and research institutions in France or abroad, or from public or private research centers.
L'archive ouverte pluridisciplinaire HAL, est destinée au dépôt et à la diffusion de documents scientifiques de niveau recherche, publiés ou non, émanant des établissements d'enseignement et de recherche français ou étrangers, des laboratoires publics ou privés. 


\title{
CAHIER de RECHERCHE
}

2005 \# 7

Efficience et Mutations des Organisations Industrielles

\section{Taille des entreprises et salaires}

\author{
GILLES MARGIRIER
}

Juin 2005

Laboratoire d'Economie de la Production et de l'Intégration Internationale - FRE2664

UPME-BP47-38040 GRENOBLE Cedex 9

lepii@upmf-grenoble.fr Tél. : 0476825692

http://www.upmf-grenoble.fr/lepii 
DOCUMENT DE TRAVAIL

\title{
Taille des entreprises et salaires
}

\author{
Gilles Margirier (\#)
}

Cette version : juin 2005

(\#) Université Pierre-Mendès France - LEPII, gilles.margirier@upmf-grenoble.fr 


\section{RESUME :}

Dans cet article, on étudie la différenciation des salaires selon la taille des entreprises en France, sur la période récente, pour une population de jeunes primo-entrants sur le marché du travail. On présente des estimations de cet écart après prise en compte de nombreuses variables de contrôle relatives aux caractéristiques de l'individu et de l'emploi occupé. Cette recherche se distingue des précédentes par une meilleure prise en compte des aptitudes individuelles filtrées par le système éducatif. On met en évidence que les différences observables dans la qualité de la main d'œuvre expliquent une part importante mais pas la totalité des écarts observés et que les caractéristiques non observables jouent un rôle important. Nous considérons aussi la possibilité d'une affectation non aléatoire des employés dans chaque classe de taille. L'hypothèse est testée à l'aide d'un modèle de sélection dans lequel l'équation de sélection est spécifiée à l'aide d'un probit ordonné. On met en évidence un biais de sélection positif pour les salariés des petites firmes et négatif pour ceux des grandes firmes. Les équations de salaires montrent que les grands établissements rémunèrent davantage les années d'étude que les petites et surtout qu'elles accordent un plus grand poids aux signaux transmis par le système éducatif via la nature des études effectuées et la délivrance ou non du diplôme.

\section{ABSTRACT :}

In this paper, we investigate the size-wage hypothesis for France, in the recent period, for young new entrants on labour market. We present estimations of the size-wage gap controlling for numerous variables representing individual and job characteristics. This research differs from the existing ones in taking account for differences in ability filtered by educational system. We show that observable differences in labour quality explain an important part, but not all the observed size-wage gap and that unobservable characteristics play a significant role. We also consider the possibility of a non-random sorting of employees within each size class. The hypothesis is tested through a selectivity-corrected wage equations model, using an ordered probit for the selection equation. A positive selection bias is found for employees in small firms and a negative one in big firms. The wages equations show that big plants pay more for each school year than the small ones and especially pay more attention to signals given by educational system via the kind of formal training and the delivery of diploma or not. 


\section{Introduction :}

Les grandes entreprises paient généralement mieux leurs salariés que les petites. Ce phénomène a été mis en évidence pour la première fois en 1911 par Moore à partir de données utilisant les salaires horaires de femmes travaillant dans l'industrie textile italienne, classés par age et taille d'établissement ${ }^{1}$. Les études empiriques menées depuis, dans de nombreux pays : USA (Brown et Medoff [1989], Davis et Haltiwanger [1996], Troske [1999]), Allemagne (Gerlach et Schmidt [1990], Schmidt et Zimmerman [1991], Criscuolo [2000]), Angleterre (Main et Reilly, [1993]), Autriche (Winter-Ebmer [1995]), Italie (Brunello et Colussi [1998]), Danemark, Finlande, Norvège, Suède (Albaek et alii [1998]), Suisse (Winter-Ebmer et Zweimuller [1999]), Canada (Lluis [2003]), Kenya et Ghana (Soderbom et alii, [2005]) convergent généralement sur ce point, même si les comparaisons internationales indiquent que les écarts mesurés par le ratio : 'salaire moyen dans les grandes firmes / salaire moyen dans les petites firmes' sont plus ou moins importants selon les pays (Oi et Idson [1999])2.

Différentes explications théoriques ont été émises au regard des faits stylisés relatifs à la relation entre salaire et taille qui relèvent de deux grands types d'explication : soit la différenciation des salaires selon la taille est due à l'utilisation d'une main d'œuvre plus productive, donc mieux rémunérée, soit les employeurs rémunèrent différemment, selon leur taille, des salariés présentant les mêmes caractéristiques ${ }^{3}$.

Selon le premier type d'explication, les grandes firmes rémunèrent leurs salariés au salaire d'équilibre concurrentiel et non au dessus (Oi et Idson [1999]). Les rémunérations versées sont plus élevées car le niveau d'effort et la productivité y sont plus forts (Idson et Oi [1999]). En conséquence elles attirent les salariés les plus productifs ${ }^{4}$. Pour autant, ces grandes firmes ne pratiquent pas des politiques spécifiques de rémunération, ce qui implique qu'un salarié d'un type donné, gagnerait le même salaire s'il travaillait dans une petite. Mais il a par contre une probabilité d'autant plus forte de travailler dans une grande unité qu'il a un niveau élevé d'aptitudes observables par l'employeur. La question essentielle devient alors celle de savoir pourquoi les grandes unités sélectionnent des salariés plus aptes et/ou pourquoi ceux-ci candidatent auprès des grandes unités ? Différents arguments peuvent être avancés : $(i)$ les grandes firmes sont précisément celles qui ont été fondées et développées par les managers les plus talentueux qui savent s'entourer eux-mêmes de salariés talentueux ; (ii) utilisant davantage de capital physique par unité de travailleur, elles font appel à des travailleurs plus qualifiés pour mettre en œuvre ce stock de capital plus important (Hammermesh [1990] ; (iii) elles recourent davantage à des technologies innovantes (informatique, électronique), d'où un appel à plus de compétences (Idson et Oi [1999]) ; (iv) les salariés sont attirés par les politiques de formation

\footnotetext{
${ }^{1}$ Voir Oi et Idson [1999] pour une présentation des données utilisées par Moore. Moore notait également la plus grande stabilité de l'emploi et la moindre durée du travail pour les salariés des plus grands établissements.

${ }^{2}$ Cf. annexe 1 pour plus d'indications sur les travaux effectués.

${ }^{3}$ Ces deux grands types d'explication sont regroupées par Brown et Medoff [1989] sous les appellations de 'labor quality explanations' (ou : 'neo-classical explanations') et de 'institutional explanations'.

${ }^{4}$ Haltiwanger et alii [1999] montrent que les firmes à plus forte productivité emploient des salariés à plus fort niveau d'éducation.
} 
que les grandes firmes développent pour mettre en œuvre ces technologies plus complexes (Barron et alii [1987], Troske [1999]) ; (v) les grandes firmes ont plus de difficultés que les petites à évaluer les performances de leurs salariés, d'où le recours à des salariés avec un plus haut niveau d'éducation pour garantir ces performances (Garen [1985]) ; (vi) elles utilisent des marchés internes pour affecter les salariés aux emplois et ont des politiques de promotion basées sur le modèle des tournois qui attirent les plus talentueux et les plus expérimentés et dissuadent les autres (vii) elles offrent de moins bonnes conditions de travail, requièrent un niveau d'effort plus élevé imposé par des rythmes plus rapides ou des responsabilités plus importantes ${ }^{5}$, ce qui implique, selon l'hypothèse néo-classique des compensations égalisatrices, des salaires plus élevés ${ }^{6}$.

Le second type d'explication suppose des politiques de rémunération différentes dans les grandes et dans les petites entreprises. Là encore, plusieurs justifications sont possibles : (i) l'existence de rentes (de monopole) reversées partiellement aux salariés; (ii) la présence de syndicats puissants qui obtiennent des salaires plus élevés pour leurs adhérents ou un salaire plus élevé que le salaire de marché pour éviter la formation de syndicats $^{7}$; (iii) des rémunérations incitatives dans les grandes firmes pour suppléer aux difficultés de contrôler l'effort des salariés (dans la lignée des théories du salaire d'efficience) ou des salaires d'embauche plus élevés pour compenser une moindre capacité à observer les aptitudes réelles des salariés (Stigler $\left.[1962]^{8}\right)$.

Cet article entend d'abord apporter des éclairages sur la situation française et la mettre au regard de la littérature relative aux autres pays. A notre connaissance, la seule étude menée sur la France est celle réalisée par Abowd, Kramarz et Margolis [1999] à partir de données longitudinales reliant des informations sur les caractéristiques des salariés à celles sur leurs employeurs respectifs sur la période 1976-1987. Décomposant l'effet brut de la taille des firmes sur le salaire en un effet propre à l'employeur et un effet propre aux caractéristiques des salariés qui la composent, les auteurs montrent que les caractéristiques des individus portent une part plus grande de l'écart de salaire selon la taille que les caractéristiques propres aux employeurs qui composent chaque catégorie de taille. Notre étude apporte des compléments à ce travail à partir des données issues d'une enquête menée en 2001 par le Cereq, auprès d'une population de nouveaux salariés, sortants du système éducatif et primo-entrants sur le marché du travail. Bien que le fichier utilisé ne résulte pas d'un appariement entre des données individuelles et des données recueillies auprès d'entreprises, il comporte des informations sur les caractéristiques personnelles et scolaires des individus enquêtés mais également sur les emplois qu'ils ont occupé

\footnotetext{
${ }^{5}$ De moins bonnes conditions de travail sont parfois considérées comme intrinsèques à la grande entreprise : celle-ci implique une plus forte division du travail, davantage de règles formelles qui, pour être supportées, doivent s'accompagner de salaires plus élevés (Masters [1969]).

${ }^{6}$ Un panorama assez complet des hypothèses théoriques liant salaires et taille a été fourni dès 1969 par Masters. Pour des analyses plus récentes, voir Brown et Medoff [1989] et Oi et Idson [1999].

${ }^{7}$ Weiss [1966] conclut à des salaires plus élevés dans les industries monopolistiques qu'il relie à la puissance des syndicat ou à la puissance de leurs menaces. Toutefois, il observe que ce résultat ne tient plus après prise en compte de la qualité de la main d'oeuvre.

8 « It is well known that wage rates are less in small plants than in large, and the difference reflects at least in part (and perhaps in whole) the lower costs to the small-scale employer of judging quality" (p 103).
} 
et sur les entreprises dans lesquelles ils ont travaillé. En outre, la personne interrogée étant amenée, lors de l'enquête, à décrire les différentes séquences d'emploi qu'elle a pu connaître au cours de la période de trois ans qui sépare sa sortie du système éducatif et le moment où elle a été enquêtée, une dimension temporelle est présente.

L'article a également pour but d'approfondir les résultats obtenus dans la littérature sur le sujet en utilisant une fonction de gains enrichie. En effet, les travaux existants étudient l'effet différencié de la taille en ajoutant les variables de taille à la fonction de gains standard proposée par Mincer où le capital humain scolaire est apprécié par le nombre d'années d'étude réalisées. Il en ressort généralement une interrogation sur la réalité de l'effet-taille et la possibilité d'aptitudes évaluées par les employeurs mais échappant à la mesure économétrique, cette évaluation pouvant différer selon la taille de l'employeur. On cherche à résoudre ce problème par l'estimation d'un modèle à effet fixe sur données de panel ou par l'estimation en différences premières de cette fonction de gains pour une population ayant changé de taille d'entreprise entre deux dates, ceci afin de neutraliser ces hétérogénéités non mesurées. L'analyse en différences premières aboutit généralement à des résultats sensiblement différents de ceux obtenus en coupe, l'élasticité du salaire à la taille étant fortement abaissée (voir Evans et Leighton [1988], Winter-Ebmer et Zweimuller [1999]). Les données utilisées dans ce travail nous permettent d'approfondir les travaux antérieurs en utilisant une fonction de gains enrichie par la prise en compte de la composante de filtre du système éducatif. On sait que cette composante de filtre joue un rôle important sur le marché du travail, les employeurs étant notamment sensibles au degré de sélectivité des formations préalablement suivies par les candidats à un emploi et à l'obtention ou non du diplôme, à durée d'études équivalente. En utilisant des variables qui différencient les individus non seulement par leur niveau d'études, mais également par le type d'établissement d'enseignement supérieur fréquenté, la spécialité suivie, l'obtention ou non du diplôme, nous montrerons que la différenciation selon la taille est fortement réduite par rapport à ce qui ressort de l'utilisation d'une fonction de gains standard.

Le troisième objectif visé dans cet article est de tester l'existence d'un effet de sélection dans la répartition des salariés selon les différentes classes de taille des établissements dans le prolongement des travaux antérieurs sur cette question (Idson et Feaster [1990), Main et Reilly [1993], Albaek et alii [1998]. Nous mettrons en évidence la présence d'un effet de sélection négatif pour les salariés des grandes unités et positif pour ceux des petites unités.

Dans la section 2, nous présentons les données et les variables utilisées et les statistiques descriptives qui y sont associées. La section 3 présente les méthodes et les résultats d'estimation. Conformément aux objectifs énoncés ci-dessus, nous estimons dans un premier temps une équation de salaire tenant compte des caractéristiques des individus et des emplois occupés en introduisant la taille des unités dans l'équation puis, dans un second temps, utilisant la dimension longitudinale des informations disponibles sur une partie de l'échantillon, nous neutralisons les hétérogénéités individuelles non observées pour mieux appréhender l'effet de la taille. Dans un troisième temps, nous présentons un modèle de sélection dans lequel les équations de salaire sont estimées séparément par classe de taille. 


\section{Données et statistiques descriptives}

Les données sont issues de l'enquête réalisée par le Céreq auprès de 55000 personnes âgées de 16 ans et plus, sorties trois ans auparavant du système éducatif et représentatives d'une cohorte de 740000 individus. L'interrogation a été réalisée au printemps 2001 et porte sur les parcours de formation antérieurs à 1998 et sur les parcours d'insertion professionnelle entre juin 1998 et mars 2001. Elle couvre l'ensemble du spectre des niveaux de formation. Limiter l'analyse aux salariés débutants réduit l'hétérogénéité des individus et notamment leur capacité différenciée à accumuler du capital humain 'on the job'. L'échantillon utilisé présente donc un avantage de ce point de vue. D'autres études ont porté sur des populations jeunes (par exemple, Evans et Leighton, [1988]). Inversement, dans la mesure où on fait l'hypothèse que cette capacité est la même pour tous les individus, elle peut être appréhendée par le temps passé au travail. Nos données présentent alors l'inconvénient de ne pas permettre d'apprécier pleinement si les grandes et les petites entreprises se différencient dans la valorisation salariale de l'expérience professionnelle. Bien que limitée (41 mois au maximum, l'acquisition éventuelle d'expérience par un travail salarié pendant les études étant négligée), l'expérience professionnelle acquise sera cependant prise en compte en cumulant les durées de tous les emplois, sauf le dernier, occupés ${ }^{9}$ par un individu depuis sa sortie du système éducatif.

Les personnes interrogées ont été amenées à décrire les différents emplois occupés depuis leur sortie du système éducatif. Les trajectoires suivies sur la période étant diverses, le nombre de séquences individuelles d'emplois enregistrées dans la base varie de 0 ( $7 \%$ des individus) à plus de 10, mais $93 \%$ ont décrit entre un et quatre emplois. L'analyse sera menée à partir des caractéristiques du dernier emploi occupé avant ou au moment de l'enquête. Pour cet emploi, les statuts et contrats de travail associés sont divers. Nous avons exclu les travailleurs indépendants et les salariés du secteur public non concurrentiel et retenu seulement ceux ayant un contrat de travail à durée déterminée ou indéterminée (CDD ou CDI), à l'exclusion des emplois avec contrat d'apprentissage, contrat de qualification et d'adaptation, des emplois intérimaires, des stages. Les salaires perçus étant connus uniquement sur une base mensuelle et le temps de travail n'étant pas connu avec précision, seuls les salariés à plein temps ont été considérés. Le fichier principal compte 26694 individus répondant à ces critères. Nous serons amenés également à utiliser un sous-fichier de 8455 individus $(31,7 \%$ ) qui avaient préalablement occupé au moins un autre emploi. Pour ce sous-échantillon, les données comportent une dimension temporelle qui s'ajoute à la dimension individuelle.

Le salaire considéré est celui perçu à l'embauche. Il est exprimé en valeur nominale et inclut la rémunération mensuelle de base et les éventuelles primes associées. La taille est appréhendée par le nombre de salariés, selon une nomenclature en six catégories : (i) de 1 à 2 , (ii) de 3 à 9 , (iii) de 10 à 49, (iv) de 50 à 199, (v) de 200 à 499, (vi) 500 et plus. On connaît également l'opinion qu'ont les salariés de leur rémunération, à travers la proportion d'individus qui estiment 'être très bien rémunérés' $(\mathrm{PAIE}=1)$.

\footnotetext{
9 Pour ce comptage, toutes les situations de travail seront considérées, quelque soit le statut juridique de l'emploi occupé.
} 
Seule la taille de l'établissement est connue mais on sait si l'entreprise de rattachement est pluri ou mono-établissement. Cette information est captée par une variable muette (PLURI=1 si l'entreprise de rattachement comporte plusieurs établissements).

La population étudiée appartient aux deux sexes ( $\mathrm{SEXE}=1$ pour hommes). L'origine sociale est connue à travers la catégorie socio-professionnelle d'appartenance du père (CSP1=1 si père « agriculteur, artisan, commerçant, chef d'entreprise », CSP2 $=1$ si père « cadre, ingénieur, technicien, profession intermédiaire », CSP3=1 si père «employé ou ouvrier »). Le capital humain d'origine scolaire est appréhendé par le nombre d'années d'études (EDUC) calculé à partir de l'âge de la personne à la fin des études (âge moins six ans). On prendra en compte également l'expérience professionnelle acquise entre la fin des études et l'emploi considéré (EXPE, exprimée en nombre de mois). Comme on l'a souligné en introduction, l'utilisation d'une fonction de gains 'à la Mincer' reposant sur la durée d'études néglige l'utilisation que font les employeurs, pour sélectionner et rémunérer les salariés, du filtrage qu'opère le système éducatif. Un enrichissement est proposé en utilisant, en complément, l'information disponible sur l'obtention ou non du diplôme correspondant au dernier cycle de formation suivi (DIPL=1 si obtention). Alternativement, on utilisera un ensemble de 43 variables muettes construites à partir d'une nomenclature couvrant l'éventail des niveaux de formations du système éducatif français, croisées avec les filières et les spécialités suivies et la possession ou non du diplôme ${ }^{10}$. Enfin, les compétences de l'individu sont également appréhendées, dans une optique schumpeterienne, par une question qui porte sur le fait de savoir si l'individu envisage ou non de se mettre un jour à son compte ${ }^{11}$. Les réponses à cette question sont traduites dans trois variables muettes $\left(\mathrm{INDEP}^{-}=1\right.$ pour une réponse négative, $\mathrm{INDEP}^{+}=1$ pour une réponse : « oui, peut être », $\mathrm{INDEP}^{++}=1$ pour une réponse : « oui, c'est dans mes projets »).

En plus du salaire perçu et de la taille de l'employeur, l'information relative à l'emploi occupé est contenue dans les variables suivantes: l'exercice de responsabilités hiérarchiques dans le poste occupé (traduit par trois variables muettes: RESP' pour « aucune responsabilité hiérarchique », $\mathrm{RESP}^{+}$pour « de 1 à 5 personnes », $\mathrm{RESP}^{++}$pour « plus de 6 personnes »), la fonction occupée (décrite à travers 11 variables; voir annexe 2), l'établissement d'accueil caractérisé (i) par son secteur d'appartenance (16 variables muettes correspondant à un découpage de l'activité selon la nomenclature NES à 16 positions), (ii) sa localisation spatiale dans un pole urbain ou rural (URBAIN=1 ou 0), et (iii) dans une des 22 régions françaises (plus l'étranger ${ }^{12}$ ). Est également connu le nombre total d'emplois (NEMP) occupés au cours des trois années couvertes par l'enquête.

\footnotetext{
${ }^{10}$ La nomenclature couvre l'ensemble des niveaux de formation (de VI à I par ordre croissant de niveau d'études). Pour les filières 'professionnelles' de niveau V à III, on distingue les spécialités industrielles, tertiaires, de santé et du secteur social. Pour l'enseignement supérieur universitaire (hors IUT), on distingue, par niveau, les spécialités 'lettres, sciences humaines, gestion', 'Maths, sciences, techniques', les concours des IUFM. Elles incluent également les écoles, en distinguant 'écoles de commerce et de gestion', 'écoles d'ingénieurs'. Chaque niveau*pécialité est dédoublé pour prendre en compte l'obtention ou non du diplôme.

${ }^{11}$ « Envisagez-vous un jour de vous mettre à votre compte (de créer votre propre entreprise, d'exercer en libéral votre profession) ?»

${ }^{12}$ Concerne les travailleurs frontaliers.
} 
Le tableau 1 présente les moyennes et écarts-type des différentes variables conditionnellement à la taille. Les salaires perçus croissent fortement avec la taille, le logarithme du salaire moyen dans les établissements de 500 salariés et plus étant de $39 \%$ supérieur à celui des établissements de 1 à 2 salariés et on note leur dispersion croissante avec la taille. Corollairement, les plus grands établissements emploient des salariés mieux éduqués et une proportion de diplômés de 11 points supérieurs à celle des plus petits ce qui va dans le sens de la distribution des salaires mais, à l'inverse de ceux-ci, l'hétérogénéité des niveaux d'éducation au sein d'une classe diminue au fur et à mesure que la taille augmente. L'analyse devra donc être approfondie en examinant la distribution des salaires non seulement conditionnellement à la taille mais aussi aux autres variables susceptibles d'affecter sa distribution.

Le souhait de travailler un jour pour son compte personnel décroît fortement avec la taille : il est émis (de manière plus ou moins affirmée) par $54 \%$ des salariés des très petites unités contre $30 \%$ seulement de ceux des très grandes unités. L'opinion sur la rémunération permet un test rudimentaire de l'hypothèse selon laquelle les différences de salaire tiennent aux politiques de salaire d'efficience plus fréquemment mises en œuvre par les grandes firmes ou de l'hypothèse équivalente émise par Stigler selon laquelle la moindre capacité des grandes firmes à déceler les qualités de leur main d'œuvre les conduit à payer davantage des salariés d'une qualité donnée. Si l'on perçoit la question qui était posée comme sous-entendant 'au regard du salaire de marché', on peut interpréter la proportion de personnes ayant une opinion très positive de leur rémunération comme celles bénéficiant de salaires d'efficience. Il ressort que ces hypothèses ne sont pas contredites par nos données dans la mesure où la proportion de très bonnes opinions sur le salaire tend à augmenter avec la taille. On notera cependant que l'augmentation n'est significative qu'au-delà de 500 salariés qui apparaît ainsi comme un seuil de démarcation du point de vue des hypothèses précédentes.

\section{Résultats d'estimation}

\subsection{Le différentiel de salaire selon la taille}

On a souligné que l'écart des salaires conditionnellement à la taille était de $39 \%$ entre les établissements de 500 salariés et plus et ceux ayant au plus 2 salariés. Il est de $11 \%$ entre les plus petites unités et celles ayant entre 10 et 49 salariés et de $28 \%$ entre ces dernières et les plus grandes.

L'hypothèse d'un recrutement différencié selon la taille amène à considérer ce qu'il advient de cet écart lorsqu'on prend en compte un certain nombre de caractéristiques individuelles. Ces caractéristiques sont observables par l'employeur lors du recrutement et dans l'activité quotidienne et lui permettent d'estimer la productivité de l'employé. Dans un premier temps, les attributs scolaires sont pris en compte selon le modèle standard de capital humain, mesurés à partir de la durée d'étude. Nous y ajoutons, outre le sexe, l'expérience acquise précédemment et le type d'aptitudes professionnelles que traduit le désir de travailler un jour pour son compte personnel. Nous constatons ainsi sans surprise (colonne 1) que ces caractéristiques observables captent une part significative de l'écart log-salarial entre les tailles, qui diminue ainsi de 7 points environ. Cependant, dans la mesure où les coefficients affectés aux variables de taille restent très 
significativement différents de 0 , il ressort que les attributs personnel et de capital humain observables ne peuvent expliquer à eux seuls la totalité de l'écart salarial constaté entre grandes et petites unités.

Comme mentionné précédemment, ce modèle mérite d'être affiné dans la mesure où les employeurs disposent d'informations plus précises sur les prétendants à l'emploi, informations qui leur sont transmises par le système éducatif, signalant les candidats selon leurs aptitudes. Ils connaissent le niveau d'études atteint mais aussi le type de connaissances particulières acquises et l'institution qui a dispensé les connaissances. Nous réestimons de ce fait l'équation précédente en remplaçant la mesure simple des aptitudes à partir du niveau d'études par celle, plus riche, des filières suivies, des spécialités de formation, du diplôme possédé ou non. Nous introduisons pour cela les variables muettes présentées précédemment. En comparant les colonnes 2 et 1, on constate que cette spécification améliore nettement la variance expliquée par le modèle et réduit fortement (de 11 points) l'écart salarial selon la taille par rapport à la mesure précédente.

Cette estimation ne prend cependant pas en compte le fait que les attributs qui sont évalués par l'employeur au moment de l'embauche le sont au regard de la fonction à exercer. L'idée sousjacente est que la productivité est moins un attribut intrinsèque de l'individu que le résultat d'un appariement satisfaisant du salarié aux tâches qui lui sont confiées (Sattinger [1993]). L'hypothèse mentionnée selon laquelle les salariés des grandes unités ont des aptitudes plus fortes doit s'entendre dans une fonction donnée et il importe donc d'en tenir compte dans l'estimation. Par ailleurs, on sait que les grandes et les petites unités sont organisées différemment. Ainsi, les premières, de par leur structure hiérarchique, offrent davantage de fonctions de supervision et de management d'équipes, ce qui peut justifier des rémunérations supérieures. La colonne 3 présente les coefficients obtenus lorsque l'on contrôle les fonctions exercées et le nombre de personnes que le salarié a sous ses ordres. Conformément à ce que l'on attendait, les coefficients des variables de taille sont à nouveau réduits, même si c'est de manière modeste.

Les coefficients obtenus pour les variables représentant les fonctions occupées (non reportés dans le tableau) nous permettent de dire quelques mots d'une hypothèse évoquée précédemment : celle de rémunérations différenciées liées aux conditions de travail. L'hypothèse de compensations égalisatrices suggère que des conditions de travail plus mauvaises sont compensées par des salaires plus élevés. Elle s'oppose à celle formulée par les théories de la segmentation du marché du travail qui considèrent qu'il existe au contraire de 'bons' emplois, cumulant de bonnes conditions de travail et des salaires élevés et des 'mauvais' emplois caractérisés au contraire par des conditions de travail dégradées et des salaires faibles. La seconde hypothèse nous semble plus conforme à la réalité et les coefficients obtenus (non reportés) pour les variables représentant les fonctions exercées vont dans ce sens. Ainsi, par exemple, la fonction de 'manutention, magasinage, transport' dont la pénibilité est avérée est bien moins rémunérée que la fonction 'production, fabrication, chantiers' (prise pour référence). Mesurer les conditions de travail est une tâche difficile et peu d'études empiriques incorporent des variables spécifiques susceptibles d'en rendre compte mais celle de Schmidt et Zimmerman [1991] va cependant dans le sens indiqué puisqu'ils observent que les salariés effectuant 'des 
tâches physiquement difficiles' ou dans un 'environnement pollué' gagnent significativement moins ${ }^{13}$.

L'étape suivante consiste à s'interroger sur ce qu'il advient du différentiel des salaires selon la taille lorsque l'on contrôle le type d'activité de l'employeur. On sait en effet que les petites entreprises sont largement présentes dans certaines activités telles que le secteur de la construction et à l'inverse totalement absentes d'autres secteurs à haute intensité capitalistique. Il est alors envisageable que l'effet taille ne reflète, pour une large part, que les différences intersectorielles de rémunération. Celles-ci capteraient notamment la dimension 'technologique' de l'écart salarial, évoquée précédemment, due aux différences de qualité de main d'œuvre du fait des différences dans l'intensité capitalistique. Elles pourraient capter également des différences dans les conditions de travail. La colonne 4 présente les résultats obtenus. L'écart selon la taille est à nouveau réduit, mais faiblement.

Enfin, on tient compte de la dimension spatiale de localisation des emplois. On sait qu'il existe des écarts salariaux selon les régions, particulièrement entre l'Ile de France et les autres régions, corrélativement à un coût de la vie plus élevé. La dimension spatiale permet de capter les écarts salariaux observés qui seraient dus à une répartition par taille inégale selon les espaces. La colonne 5 permet de constater que la réduction apportée aux coefficients de la taille par la dimension spatiale est plus marquée que celle due à la prise en compte des différences intersectorielles.

Dans la colonne 6, les variables muettes de taille sont remplacées par une seule variable pseudocontinue construite en considérant les centres des classes ${ }^{14}$. L'étude détaillée menée par Albaeck et alii (1998) concernant les erreurs de mesure qui découlent d'une telle approximation les a amenés à conclure à la validité d'une telle démarche ${ }^{15}$. On estime ainsi à 0,022 l'élasticité du salaire à la variation de l'effectif. Cette estimation est à mettre en regard de celles obtenues par Albaeck et alii [1998] qui vont de 0,020 pour la Finlande à 0,025 pour la Norvège et avec celle de 0,027 obtenue par Brown et Medoff [1989] pour les USA ${ }^{16}$. La comparaison doit cependant tenir compte du fait que les spécifications retenues dans ces études mesurent le capital humain à partir du nombre d'années d'étude, ce qui majore le coefficient d'élasticité (si on reprend l'estimation présentée à la colonne 6 en remplaçant les variables de spécialité de formation par le nombre d'années d'études pour être plus proche des travaux cités, on arrive à une élasticité de $0,030)$.

\footnotetext{
${ }^{13}$ Les tests effectués par Brown et Medoff [1989] ainsi que par Albaek et alii [1998] les laissent également sceptiques sur la portée explicative des conditions de travail sur la relation salaire-taille.

${ }^{14}$ Pour la dernière classe, la valeur 1500 sera retenue à l'instar de Albaeck et alii (cf note suivante). Voir également Brown et Medoff [1989] (table 1) pour une démarche identique.

15 Les auteurs disposent de la taille réelle des établissements. Cette information est utilisée pour affecter les établissements à six classes de taille pour lesquelles sont ensuite considérés les centres de classe (la valeur 1500 est retenue pour la dernière classe). Les tests de validation effectués les conduisent à conclure que "the use of mid-points seems to be an innocent procedure and produces similar results as more exact measures of employer size" (p 441).

${ }^{16}$ Egalement avec des variables de contrôle proches de celles utilisées ici, à savoir : sexe, syndiqué, race, éducation, expérience, (expérience) $)^{2}$, régions (3 variables), secteurs (41 variables), fonctions (8 variables).
} 
Les estimations précédentes recourent à la taille des établissements. Toutefois, deux établissements de taille identique peuvent appartenir à des firmes de taille très différente. Les agences bancaires, par exemple, sont généralement de petite taille alors qu'elles appartiennent à des firmes de grande taille ${ }^{17}$. Dans la littérature, les explications 'technologiques' renvoient plutôt à la taille de l'établissement qu'à celles de la firme. Il en est ainsi de l'hypothèse évoquée selon laquelle un salaire plus élevé compense de moins bonnes conditions de travail dans les grandes unités ou de l'hypothèse selon laquelle elles imposent des rythmes de travail plus élevés et utilisent des équipements plus lourds et plus coûteux. Inversement, les explications 'institutionnelles' faisant appel au rôle des syndicats ou au pouvoir de marché renvoient plus naturellement à la taille de la firme qu'à celle de l'unité de production. Nous avons évoqué également l'hypothèse d'une moindre capacité des grandes firmes à superviser leurs salariés ce qui les amène à proposer des salaires plus élevés pour attirer ceux ayant les plus fortes motivations et la plus forte productivité. La plupart des travaux empiriques utilisent soit des données relatives à la taille de l'établissement, soit à celle de l'entreprise même si quelques travaux incluent les deux ${ }^{18}$. L'introduction de termes d'interaction entre la taille de l'établissement et l'appartenance à une firme mono ou pluri-établissements permet d'y voir un peu plus clair sur cette question. Les résultats présentés colonnes 7 et 8 confirment l'effet spécifique de la taille de l'entreprise sur le salaire, venant s'ajouter à l'effet de la taille de l'établissement. La colonne 7 reprend la spécification présentée en colonne 5 et la colonne 8 celle de la colonne 7 mais en incluant l'effet taille de l'entreprise. On constate, colonne 8, que le fait d'appartenir à une firme pluri-établissement apporte un bonus salarial. Les termes d'interaction de la colonne 7 montrent que ce bonus salarial se vérifie surtout pour les salariés des établissements de plus de 200 salariés.

Les coefficients des termes d'interaction sont positifs, traduisant le fait que, toutes choses égales par ailleurs et notamment à taille d'établissement égale, un salarié appartenant à une plus grande firme, pluri-établissements, sera mieux rémunéré. Cet effet de la taille de l'entreprise est assez fort pour les salariés des petits établissements puisque le fait d'appartenir à une entreprise pluri-établissements apporte un bonus log-salarial de 2,2\%, supérieur à celui apporté par la taille de l'établissement. L'effet est peu sensible pour les salariés des établissements de taille moyenne et redevient important pour ceux des grands établissements, mais l'effet de la taille de l'entreprise y est moindre que celui de la taille des établissements.

\footnotetext{
${ }^{17}$ Dans notre échantillon, $54 \%$ des individus interrogés travaillent dans des firmes composées de plusieurs unités. Le tableau 1 montre que cette proportion est fortement croissante avec la taille de l'établissement : $21,2 \%$ de ceux travaillant dans les plus petits établissements sont salariés d'un ensemble plus vaste, contre $83,3 \%$ de ceux travaillant dans les plus grands. Autrement dit, la plupart de ceux travaillant dans un petit établissement sont en contact direct avec leur employeur, ce qui n'est pas le cas pour ceux qui travaillent dans des unités plus grandes.

${ }^{18}$ Voir annexe 1. Parmi les travaux qui utilisent des données portant à la fois sur la taille de l'établissement et celle de l'entreprise, Mellow [1982] conclut à un double impact sur le salaire mais Evans et Leighton [1988] concluent qu'une fois contrôlée la taille de la firme, la taille des établissements n'a un effet significatif que si elle est élevée (plus de 1000 salariés).
} 


\subsection{L'impact des aptitudes non mesurées}

On a vu que l'estimation d'une équation de gains considérant que les employeurs utilisent au mieux les signaux transmis par le système éducatif permettait d'abaisser fortement l'écart salarial selon la taille par rapport à la formulation standard utilisée dans la plupart des études. La question des aptitudes non évaluées économétriquement a donc reçu un début de réponse. On peut cependant s'interroger sur la part qui demeure incluse dans les coefficients affectés aux classes de taille dans notre estimation mais qui pourtant ont été évaluées par les employeurs lors des entretiens d'embauche et qui peuvent justifier une partie de la dispersion des salaires ${ }^{19}$.

La question des différences de qualité de la main d'œuvre dans les grandes et les petites unités reste donc ouverte. Il est cependant possible d'en apprendre un peu plus en considérant, pour des individus ayant changé d'emploi et éventuellement à cette occasion de taille d'établissement, la variation de salaire qui en a découlé. Considérons en effet l'équation suivante qui a servi aux estimations précédentes :

$$
w_{i t}=X_{i t} \beta+Z_{i t} \gamma+\sum_{j} \delta_{j} C_{i j t}+\varepsilon_{i t}
$$

où $w_{i t}$ désigne le logarithme du salaire de l'individu $i$ associé à l'emploi considéré à la date $t, X_{i t}$ un ensemble de caractéristiques personnelles observées, $Z_{i t}$ un ensemble d'attributs observés de l'emploi occupé, $\beta$ et $\gamma$ les vecteurs des paramètres associés, $C_{i j t}$ une variable indicatrice de la classe de taille $j$ dans lequel l'individu travaille à la date $t, \delta_{j}$ les paramètres associés, $\varepsilon_{i t}$ un terme de perturbation. Supposons que ce terme de perturbation se décompose de la manière suivante :

$$
\varepsilon_{i t}=\eta_{i}+\xi_{i t}
$$

avec $\eta_{i}$ un terme constant dans le temps, traduisant des aptitudes individuelles observables par l'employeur mais non observées économétriquement et $\xi_{i t}$ un terme purement aléatoire.

Considérons ensuite la variation des gains perçus pour l'emploi occupé à la date $t$ et pour le précédent, occupé à la date $(t-1)$. Si les différences de qualité de main d'œuvre recrutée expliquent une part de l'écart constaté entre grandes et petites unités, alors le changement de taille ne devrait apporter aucun supplément salarial, en contrôlant bien entendu les changements dans les caractéristiques de l'emploi occupé. Si tel n'est pas le cas, et à condition que ces qualités soient rémunérées de manière identique par tous les employeurs, cela laisse entendre, qu'à qualité équivalente et emploi équivalent, la rémunération est plus élevée dans les grandes unités. Pour autant, cette déduction ne tient que s'il est possible économétriquement d'observer parfaitement les différences de qualité de main d'œuvre. Si ce n'est pas le cas, il est possible que grands et petits employeurs emploient réellement des salariés de qualité différente, qu'ils observent comme tels et qu'ils rémunèrent différemment de ce fait.

La variation des gains est exprimée par l'équation suivante, déduite de (1) :

\footnotetext{
${ }^{19}$ Barron et alii [1987] concluent que les grands employeurs examinent davantage de candidatures par poste vacant que les petits. Par contre, ils n'observent pas un temps d'examen significativement plus long pour chaque candidature.
} 


$$
w_{i t}-w_{i, t-1}=\left(X_{i t}-X_{i, t-1}\right) \beta+\left(Z_{i t}-Z_{i, t-1}\right) \gamma+\sum_{j} \delta_{j}\left(C_{i j t}-C_{i j, t-1}\right)+\xi_{i t}-\xi_{i, t-1}
$$

Cette équation élimine les caractéristiques observées qui sont constantes dans le temps ainsi que les aptitudes non observées. Demeurent les caractéristiques personnelles observées et les attributs de l'emploi qui ont varié entre $(t-1)$ et $t$. L'explication par les différences de qualité suppose que les coefficients $\delta_{j}$ ne diffèrent pas significativement entre eux. Nous estimons l'équation précédente à partir du sous-ensemble des individus ayant occupé au moins deux emplois $^{20}$ et retenons les caractéristiques du premier emploi et du dernier emploi occupés. Les résultats sont présentés au tableau 3.

Les deux premières colonnes reproduisent sur l'échantillon concerné les estimations en coupe présentées au tableau 2, colonnes 5 et 6 . La colonne 3 présente les résultats de l'estimation en différences premières issus de l'équation (2) et la colonne 4 reproduit cette estimation, les classes de taille étant remplacées par la taille estimée. La variable dépendante est le taux de variation du salaire. Associé au changement d'emploi, ce taux de variation s'explique par l'acquisition d'expérience professionnelle, par une augmentation éventuelle des responsabilités hiérarchiques assumées, par les changements éventuels de fonction exercée, de secteur d'activité, de localisation géographique et, bien entendu, par un changement de taille d'établissement. On constate qu'il demeure un écart substantiel entre les plus grandes unités et les plus petites, puisque le modèle prédit que passer d'une très petite à une très grande unité majore de $9,2 \%$ le taux de croissance du salaire. En utilisant les résultats obtenus à l'aide de la mesure continue de la taille (colonne 4), on peut calculer qu'un salarié passant d'un établissement d'une taille d'un écart-type inférieur à la moyenne à un établissement d'une taille d'un écart-type supérieur obtient un salaire supérieur de $5,7 \%$ au précédent.

L'explication par les différences d'aptitudes n'est donc pas entièrement confortée par ces résultats et on ne peut exclure l'hypothèse d'une rémunération différenciée de salariés identiques par les grands et les petits employeurs. Cette conclusion est similaire à celle formulée par Brown et Medoff [1989]. Malgré tout, la comparaison des résultats obtenus aux colonnes 3 et 4 avec ceux des colonnes 1 et 2 fait ressortir une différence notable dans les écarts entre les coefficients associés à la taille. L'élasticité, qui est de 0,0195 dans l'estimation en coupe est ramenée à 0,0139 par l'estimation en différences premières. L'inclusion dans l'effet taille de ce qui relève en réalité de l'hétérogénéité individuelle non observée aboutit donc à surestimer cet effet de $40 \%$. Ces hétérogénéités individuelles expliquent donc une part importante des écarts constatés dans les

\footnotetext{
${ }^{20}$ Parmi eux, 38,2\% ont changé d'emploi sans changer de classe de taille d'établissement, 35,6 \% sont allés dans des établissements de plus grande taille et 26,2\% dans des établissements de taille plus petite. Tout changement de classe de taille est supposé être associé à un changement d'entreprise (établissement). En réalité, il est envisageable que certains de ces changements soient fictifs : les deux séquences d'emploi enregistrées pourraient se situer dans la même entreprise, matérialisées par une coupure entre les deux séquences. Dans ce cas, il est envisageable que l'entreprise ait changé de taille entre les deux périodes ce qui serait, à tort, enregistré comme une migration de l'individu vers une entreprise différente, de taille différente. Nous avons vérifié que le retour dans la même entreprise ne concernait qu'un très faible nombre d'individus $(0,5 \%)$.
} 
estimations en coupe instantanée ${ }^{21}$, même si l'amélioration de la fonction de gains a permis de la réduire nettement ${ }^{22}$.

Pour compléter l'analyse, on a regroupé les variables de contrôle pour les changements de fonction, de secteur et de région dans trois variables prenant la valeur 1 si la fonction occupée n'est plus la même, si l'emploi n'est plus dans le même secteur d'activité, s'il n'est plus situé dans la même région. La colonne 5 présente les résultats. On constate l'effet positif de la mobilité géographique sur la variation du salaire (Margirier [2005]). A l'inverse, le changement de secteur d'activité et le changement de fonctions n'exercent aucun effet significatif.

\subsection{Un test de l'effet de sélection}

Les développements précédents ont permis de faire la part dans la dispersion des salaires selon la taille, de ce qui relève de différences dans les qualités de main d'œuvre utilisée et de ce qui peut relever de politiques de rémunérations différenciées. On a vu que les différences de qualité de main d'œuvre semblaient à elles seules ne pas pouvoir expliquer la totalité des écarts salariaux, donnant un certain crédit aux explications 'institutionnelles'.

Ceci milite pour l'estimation de fonctions de gains séparées par classe de taille afin d'apprécier les rendements différenciés des différentes caractéristiques. Une condition requise pour une estimation sans biais des équations de salaires par les MCO est que l'échantillon soit constitué de manière aléatoire au sein de la population d'individus identiques au plan de l'observation. Cette condition n'est cependant pas forcément remplie. Il est possible que des individus à caractéristiques observables identiques se répartissent selon les tailles en fonction de critères non pris en compte dans les équations de salaires mais étant propres à chacun et déterminant leur propre utilité à travailler dans une unité d'une taille donnée et l'utilité pour celle-ci de le recruter plutôt qu'un autre. On considère que cette utilité dépend d'un vecteur de caractéristiques observables et d'une composante stochastique incluant des caractéristiques non observables : $U_{i}^{*}=U\left(B_{i}, v_{i}\right)$. L'endogénéisation de la variable 'taille' passe par l'estimation d'un modèle avec biais de sélection (Heckman [1979]). Idson et Feaster [1990] ont estimé un tel modèle pour la relation taille-salaire aux USA ainsi que Main et Reilly [1993] pour le RoyaumeUni, en recourant à un probit ordonné pour l'estimation de l'équation de sélection. Nous procédons de même ici.

En désignant par X les caractéristiques individuelles, Z celles de l'emploi occupé, $j$ les différentes classes de taille, les équations de salaire s'écrivent :

$$
w_{i j}=X_{i} \beta_{j}+Z_{i} \gamma_{j}+u_{i j} \quad u_{i j} \sim N\left(0, \sigma_{u_{j}}^{2}\right)
$$

auxquelles on ajoute une équation exprimant l'utilité individuelle de travailler pour une entreprise d'une classe de taille donnée, soit :

\footnotetext{
${ }^{21}$ On doit noter que les erreurs de mesure tendent à abaisser davantage les coefficients obtenus sur les estimations longitudinales que sur les estimations en coupe. Voir sur ce point Griliches et Hausman [1986].

${ }^{22}$ En utilisant une fonction de gains 'standard', l'élasticité mesurée en coupe sur l'échantillon restreint à ceux ayant changé d'emploi est de 0,0284 au lieu de 0,0195. Du coup, la surestimation est de $104 \%(0,0284 / 0,0139-1)$.
} 


$$
U_{i}^{*}=B_{i} \delta+v_{i}
$$

$U_{i}^{*}$ est une variable latente non observable. $B_{i}$ contient les variables explicatives du choix de la taille et $v_{i t}$ est un terme d'erreur $\left(v_{i} \sim N(0,1)\right)^{23}$ corrélé aux termes $u_{j}\left(\rho_{u_{j} v} \neq 0\right) . U_{i}^{*}$ prend des valeurs de seuil $\mu$ auxquelles on fait correspondre les différentes classes de taille. Pour alléger l'estimation et la présentation des résultats, nous retenons quatre classes de taille (1-9, 10-49, 50-199, 200 et + ) au lieu des six précédentes. Elles sont définies par les variables binaires $C_{j=0, \ldots, 3}$ prenant la valeur 1 si $C_{i}=j, 0$ sinon. Plus précisément, si :

$$
\begin{array}{ll}
U_{i}^{*}<\mu_{0} & \text { on observe } C_{i 0}=1,0 \text { sinon, } \\
\mu_{0} \leq U_{i}^{*}<\mu_{1} & \text { on observe } C_{i 1}=1,0 \text { sinon. } \\
\mu_{1} \leq U_{i}^{*}<\mu_{2} & \text { on observe } C_{i 2}=1,0 \text { sinon. } \\
U_{i}^{*} \geq \mu_{2} & \text { on observe } C_{i 3}=1,0 \text { sinon. }
\end{array}
$$

Compte tenu du biais de sélection, les espérances conditionnelles des salaires sont (en posant $\left.\mu_{0}=0\right)$ :

$$
\begin{aligned}
& E\left(w_{i 0}\right)=X_{i} \beta_{0}+Z_{i} \gamma_{0}+E\left(u_{i 0} \mid v_{i}<-B_{i} \delta\right) \\
& E\left(w_{i 1}\right)=X_{i} \beta_{1}+Z_{i} \gamma_{1}+E\left(u_{i 1} \mid-B_{i} \delta \leq v_{i}<\mu_{1}-B_{i} \delta\right) \\
& E\left(w_{i 2}\right)=X_{i} \beta_{2}+Z_{i} \gamma_{2}+E\left(u_{i 2} \mid \mu_{1}-B_{i} \delta \leq v_{i}<\mu_{2}-B_{i} \delta\right) \\
& E\left(w_{i 3}\right)=X_{i} \beta_{3}+Z_{i} \gamma_{3}+E\left(u_{i 3} \mid v_{i} \geq \mu_{2}-B_{i} \delta\right)
\end{aligned}
$$

Les équations de salaire prenant en compte l'effet de sélection s'écrivent alors :

$$
\begin{aligned}
w_{i 0}= & X_{i} \beta_{0}+Z_{i} \gamma_{0}+\rho_{u_{0} v} \sigma_{0}\left\{\left[-\phi\left(B_{i} \delta\right)\right] /\left[1-\Phi\left(B_{i} \delta\right)\right]\right\}+\zeta_{i 0} \\
w_{i 1}= & X_{i} \beta_{1}+Z_{i} \gamma_{1} \\
& +\rho_{u_{1} v} \sigma_{1}\left\{\left[\phi\left(-B_{i} \delta\right)-\phi\left(\mu_{1}-B_{i} \delta\right)\right] /\left[\Phi\left(\mu_{1}-B_{i} \delta\right)-\Phi\left(-B_{i} \delta\right)\right]\right\}+\zeta_{i 1} \\
w_{i 2}= & X_{i} \beta_{2}+Z_{i} \gamma_{2} \\
& +\rho_{u_{2} v} \rho_{2 v} \sigma_{2}\left\{\left[\phi\left(\mu_{2}-B_{i} \delta\right)-\phi\left(\mu_{1}-B_{i} \delta\right)\right] /\left[\Phi\left(\mu_{2}-B_{i} \delta\right)-\Phi\left(\mu_{1}-B_{i} \delta\right)\right]\right\}+\zeta_{i 2} \\
w_{i 3}= & X_{i} \beta_{3}+Z_{i} \gamma_{3}+\rho_{u_{3} v} \sigma_{3}\left\{\phi\left(\mu_{2}-B_{i} \delta\right) /\left[1-\Phi\left(\mu_{2}-B_{i} \delta\right)\right]\right\}+\zeta_{i 3}
\end{aligned}
$$

avec $\phi$ et $\Phi$ respectivement la densité de probabilité et la fonction de répartition de la loi normale, $\zeta_{i j}$ un terme de perturbation.

Les valeurs $\mu$ et le vecteur des paramètres $\delta$ peuvent être obtenus par maximum de vraisemblance à l'aide d'un probit ordonné ${ }^{24}$. Les inverses des ratios de Mills sont ensuite

\footnotetext{
${ }^{23}$ Les valeurs prises par $\mathrm{U}^{*}$ étant inconnues, la variance est normalisée à 1.

${ }^{24}$ La fonction de log-vraisemblance est $: L=\prod_{i=1}^{n} \prod_{j=0}^{J-1}\left[\Phi\left(\mu_{j}-B_{i} \delta\right)-\Phi\left(\mu_{j-1}-B_{i} \delta\right)\right]^{C_{i j}}$.
} 
calculées et définissent une nouvelle variable, représentant l'effet de sélection, introduite dans les équations de salaires, ces dernières étant estimées à l'aide des MCO. Les valeurs $\lambda_{j}=\rho_{u_{j} v} \sigma_{j}$ sont les coefficients attachés à la variable de sélection. Ce sont des paramètres à estimer avec les coefficients $\beta_{j}$ et $\gamma_{j}$ des autres variables.

Les résultats du Probit ordonné sont présentés dans le tableau 4. Dans une optique de comparaison, le choix des variables s’inspire des spécifications retenues dans les études du même type. On constate que la probabilité de travailler dans une grande unité augmente avec le niveau d'études. A été pris en compte également, le nombre de séquences d'emploi préalables comme indicateur de comportement. L'idée est ici qu'une plus forte rotation sur les emplois implique une préférence pour les petites unités où les emplois sont moins stables (Evans et Leighton [1988]), ce qui se vérifie par le résultat obtenu. Toujours au regard d'un critère de stabilité, on fait l'hypothèse que la vie en couple est susceptible d'induire une préférence pour les unités de grande taille qui apportent plus de stabilité de l'emploi (Evans et Leighton [1988]), Idson et Feaster [1990]). Le signe est également conforme aux attentes mais le coefficient n'est pas significatif. L'origine sociale est supposée induire des préférences différenciées : les enfants d'artisans, agriculteurs, commerçants, chefs d'entreprise sont susceptibles d'être plus nombreux à intégrer des unités de petite taille que ceux des autres catégories. Le résultat obtenu confirme cette hypothèse. Nous disposons également d'une variable clé, absente dans les travaux cités, qui est le fait de déclarer vouloir ou non travailler un jour à son compte. Ceux ayant répondu positivement à cette question sont attendus préférer les petites unités aux grandes. Les résultats d'estimation confirment cette préférence : plus la volonté de travailler à son compte est affirmée, plus la probabilité de travailler dans une petite unité est forte. Enfin, les valeurs des seuils $\mu_{1}$ et $\mu_{2}$ indiquent que les facteurs inobservables participent à la sélection et que ceux qui ont les valeurs les plus élevées ont une probabilité plus forte de travailler dans une grande unité.

Les résultats de l'estimation des équations de gains par classes de taille sont présentés au tableau 5. Dans un souci de concision, on a fait figurer uniquement les classes de taille extrêmes. Les colonnes 1 et 2 présentent les résultats obtenus par les MCO non corrigés de l'effet de sélection. Les colonnes 3 et 4 reprennent l'estimation en incluant l'effet de sélection. L'hypothèse d'une répartition non aléatoire des individus dans les différentes classes de taille est validée par le niveau des coefficients obtenus pour la variable de sélection $(\lambda)$. Ces coefficients sont significativement différents de 0 . Le coefficient négatif pour les unités de petite taille indique un effet de sélection positif (voir équation 7a) traduisant un salaire prédit, connaissant la taille de l'employeur (salaire conditionnel), supérieur au salaire prédit ne connaissant pas cette taille (salaire non conditionnel) et le fait que les individus employés dans ce type d'établissement gagnent plus, en moyenne, que le gain moyen d'individus qui auraient été affectés aléatoirement à cette classe de taille. A l'inverse, on observe un coefficient négatif traduisant un effet de sélection négatif (voir équation 7d) pour les employés d'unités de grande taille traduisant un salaire prédit conditionnellement à la taille, inférieur au salaire non conditionnel. On peut s'interroger sur la robustesse des résultats obtenus. Nous avons testé différentes spécifications pour l'équation de sélection sans que les résultats soient significativement modifiés. Ces résultats 
sont identiques à ceux obtenus par Idson et Feaster $[1990]^{25}$. Ils y voient l'effet de la préférence différenciée pour le travail indépendant, variable non observable dans leur modèle et qui affecterait simultanément la probabilité de choix de la taille et le salaire via les termes de perturbation et selon le mécanisme suivant : une influence négative sur le choix des unités de grande taille, une influence positive sur les salaires payés par les entreprises de toutes tailles (car ces aptitudes laissent présager une productivité supérieure dans tous les contextes), la covariance négative entre les termes de perturbation étant traduite par des coefficients négatifs pour la variable de sélection dans toutes les classes de taille. Ces salariés à forte productivité se dirigeant de préférence vers les petites unités, ceci a pour conséquence, dans les unités de grande taille, un salaire moyen inférieur à celui que l'on aurait observé si la répartition avait été aléatoire (et inversement dans les petites unités). Le processus de sélection a donc pour conséquence de réduire le différentiel de salaires entre les grandes et les petites unités. A la différence de celles des auteurs cités, nos données nous ont permis d'observer la préférence pour le travail indépendant. Leur raisonnement se trouve confirmé par nos résultats : on observe que la préférence pour le travail indépendant augmente la probabilité de travailler dans une petite unité (coefficient négatif dans l'équation de sélection ; tableau 4) et accroît le salaire perçu dans toutes les classes de taille (les coefficients de INDEP + et INDEP ++ sont positifs et plus forts pour INDEP ++ que pour INDEP + dans les équations de salaire; tableau 5 , col. 3 et 4). Cependant, si telle était l'explication, l'effet de sélection ne devrait pas apparaître dans nos estimations puisque le critère de préférence pour le travail indépendant est observable. Le fait qu'il demeure montre qu'il existe d'autres processus à l'œuvre.

Nous avons testé plusieurs spécifications alternatives du modèle complet, qui aboutissent aux observations suivantes : (i) réestimer le modèle en ôtant ce critère de l'équation de sélection et des équations de salaire ${ }^{26}$ ou alors (ii) l'ôter de l'équation de sélection en le conservant dans les équations de salaires ${ }^{27}$ laisse intacte la mise en évidence d'un effet de sélection ; par contre (iii) l'inclure dans l'équation de sélection mais considérer qu'il n'est pas perçu par les employeurs comme un signal positif et rémunéré en tant que tel tend à faire disparaître l'effet de sélection (il demeure significatif et toujours négatif dans les grandes unités mais est fortement atténué et il devient non significatif dans les petites). Le choix de la spécification a donc toute son importance dans les résultats obtenus mais celle que nous avons présenté nous semble la plus pertinente. On notera encore à travers la comparaison des estimations obtenues aux colonnes, respectivement 1 et 3,2 et 4 , que la correction pour biais de sélection affecte beaucoup les coefficients des variables traduisant la préférence pour le travail indépendant. Enfin, il ressort que si les entreprises de toutes tailles rémunèrent spécifiquement les aptitudes au travail indépendant, celles-ci le sont légèrement plus dans les petites unités que dans les grandes.

\footnotetext{
25 Main et Reilly [1993] n'observent aucun effet de sélection dans leurs estimations. Albaek et alii [1998] testent l'endogénéité de la taille (variable continue) en utilisant les doubles moindres carrés. Ils concluent à une élasticité plus forte après prise en compte de l'effet de sélection dans trois pays sur quatre, soit un effet de sélection négatif.

${ }^{26}$ Spécification correspondant à celle testée par Idson et Feaster [1990].

${ }^{27}$ Mais l'hypothèse sous-jacente selon laquelle cela n'aurait pas d'influence sur le choix de la taille n'est pas très satisfaisante.
} 
Concernant les rendements différenciés de l'éducation, nous avons réestimé le modèle de sélection en reprenant la spécification courante consistant à introduire le nombre d'années d'étude. Nous y avons ajouté toutefois la possession ou non du diplôme pour prendre en compte le filtrage. Les résultats sont présentés aux colonnes 4 et 5 . On constate d'une part que les coefficients de la variable 'diplôme' sont très élevés (l'obtention du diplôme 'vaut' entre 2,5 et 5 années d'études) ce qui témoigne de l'intérêt d'en tenir compte dans les équations de salaire et d'autre part qu'ils sont fortement différenciés selon la taille et plus de deux fois plus élevés dans les grandes unités que dans les petites. Ainsi, les firmes se différencient moins, selon leur taille, par la rémunération des années d'études (20\% d'écart dans les taux de rendements) que par l'importance accordée aux diplômes et les grandes firmes s'appuient davantage sur le filtrage opéré par le système scolaire que les petites. Ce résultat souligne a contrario que l'hypothèse selon laquelle les grandes entreprises ont une moindre possibilité d'évaluer leurs salariés que les petites ou des coûts plus élevés pour le faire (voir Garen [1985] sur ce point) néglige précisément la possibilité d'utiliser cette fonction du système éducatif et montre que les grandes firmes l'utilisent intensément pour compenser ce handicap.

Pour le reste, on notera que les écarts de salaire entre hommes et femmes sont plus grands dans les petites unités, que les gains de l'expérience sont plus progressifs dans les grandes unité et que la préférence pour le travail indépendant semble recouvrir des qualités appréciées non seulement par les petites mais également par les grandes unités. Enfin, les variables retenues expliquent une part nettement plus importante de la dispersion des salaires dans les grandes unités (48 à $58 \%$ ) que dans les petites (30 à $35 \%$ ). La détermination des rémunérations dans ces dernières obéit donc moins aux critères 'objectifs' que l'économétrie prend généralement en compte.

\section{Conclusion}

Cet article a analysé la différenciation des salaires selon la taille des établissements en France sur la période récente pour une population de jeunes, primo-entrants sur le marché du travail. Il montre que l'insertion professionnelle se fait le plus souvent en passant progressivement d'un établissement de petite taille vers un plus grand. Une raison à cela est l'écart de rémunération qui les caractérise. Mesuré sur des données en coupe, cet écart est de $13 \%$ environ entre les unités de 1 à 2 salariés et celles de plus de 500 salariés et l'élasticité du salaire à la taille de l'établissement est de l'ordre de 0,022. L'élimination de caractéristiques non observables par une estimation de la variation du salaire imputable à un changement de taille d'établissement à l'occasion d'un changement d'emploi laisse persister un écart de $9 \%$ environ entre les plus petites et les plus grandes unités. L'hétérogénéité individuelle ne peut donc expliquer la totalité des écarts constatés et l'explication 'institutionnelle' de ces écarts a une certaine validité.

L'estimation d'un modèle adéquat a permis de mettre en évidence la présence d'un effet de sélection négatif pour les grandes unités et positif pour les petites. L'intérêt du résultat obtenu est renforcé par le fait que contrairement aux études antérieures, nous avons pu prendre en compte explicitement le souhait ou non de travailler un jour pour son propre compte, considéré comme une proxi d'aptitudes non révélées par le système scolaire et susceptibles d'influer sur le choix d'une taille d'établissement. Cette variable apparaît comme une dimension importante à 
prendre en compte dans les études de ce type. Le modèle a montré par ailleurs que la probabilité de travailler dans une grande unité était plus élevée pour les plus éduqués. Enfin, il ressort qu'une année d'étude est mieux rémunérée dans une grande unité que dans une petite mais l'écart est surtout important dans la considération du diplôme, les grandes unités discriminant beaucoup plus leurs salariés selon ce critère que les petites. 


\section{Références}

Abowd J. M., Kramarz F., Margolis D.N. [1999], "High Wage Workers and High Wage Firms", Econometrica, vol. 67, $\mathrm{n}^{\circ} 2,251-333$.

Albaek K., Arai M., Asplund R., Barth E., Strojer Madsen E. [1998], "Measuring Wage effects of Plant Size", Labour Economics, vol.5, n4 4, 425-448.

Barron J.M., Black D.A., Loewenstein [1987], "Employer Size: The Implications for Search, Training, Capital Investment, Starting Wages, and Wage Growth", Journal of Labor Economics, vol 5, $\mathrm{n}^{\circ} 1,76-89$.

Brown C., Medoff J. [1989], "The Employer Size-Wage Effect”, Journal of Political Economy, vol. $97, \mathrm{n}^{\circ} 3,1027-1059$.

Brunello G., Colussi A. [1998], "The Employer Size-Wage Effect: Evidence From Italy", Labour Economics, vol. 5, $\mathrm{n}^{\circ} 2,217-230$.

Criscuolo C. [2000], "Employer Size-Wage Effect: A Critical Review and an Econometric Analysis", working paper $\mathrm{n}^{\circ} 277$, Universita degli studi di Siena, Dipartimento di economia politica, janvier, 41 pages.

Davis S.J., Haltiwanger J. [1996], "Employer Size and the Wage Structure in the U.S. Manufacturing “, Annales d'Economie et de Statistiques, $n^{\circ}$ 41/42, 323-368.

Evans D.S., Leighton L.S. [1988], "Why Do Smaller Firms Pay Less ?", The Journal of Human Resources, vol. XXIV, $\mathrm{n}^{\circ} 2,299-317$.

Garen J.E. [1985], "Worker Heterogeneity, Job screening, and Firm Size", Journal of Political Economy, vol. 93, $\mathrm{n}^{\circ} 4,715-739$.

Gerlach K., Schmidt E.M. [1990], "Firm Size and Wages", Labour, vol. 4, n², 27-50.

Griliches Z., Hausman J.A. [1986], "Errors in variable in panel data", Journal of Econometrics, vol 31, 93-118.

Haltiwanger J.C., Lane J.I., Spletzer J.R. [1999], "Productivity Differences across Employers: The Role of Employer Size, Age and Human Capital", American Economic Review, vol. $89, \mathrm{n}^{\circ} 2,94-98$.

Hammermesh D.S. [1990], "Commentary", The Economics of Firm Size, Market Structure, and Social Performance, J.J. Siegfried ed., Washington, Fed. Trade Commission.

Heckman J.J. [1979], "Sample Selection as a Specification Error", Econometrica, vol. 47 (1), $153-161$.

Idson T. L., Feaster D.J. [1990], "A Selectivity Model of Employer Size-Wage Differentials", Journal of Labor Economics, vol. 8, $\mathrm{n}^{\circ} 1,99-122$.

Idson T.L., Oi W.Y., [1999], "Workers Are More Productive in Large Firms", American Economic Review, vol. 89, $\mathrm{n}^{\circ} 2,104-108$. 
Lluis S. [2003], "Endogeneous Choice of Firm Size and the Structure of Wages: A Comparison of Canada and the United States", Working paper, University of Minnesota, 34 pages.

Main B.G., Reilly B. [1993], "The Employer Size-Wage Gap: Evidence for Britain", Economica, vol. $60,125-142$.

Margirier G. [2005], "Mobilité géographique et salaires à l'entrée sur le marché du travail", document de travail, Université Pierre Mendès France, Lepii, 21 pages.

Masters S.H. [1969], "An Interindustry Analysis of Wages and Plant Size", The Review of Economics and Statistics, vol. 51, n 3, 341-345.

Mellow W. [1982], "Employer Size and Wages", The Review of Economic and Statistics, vol. $64, n^{\circ} 3,495-501$.

Moore H.L. [1911], Laws of Wages: An Essay in Statistical Economics, A.M. Kelley, New York (réédition 1967).

Oi W.Y., Idson T.L. [1999], "Firm Size and Wages" in: Handbook of Labour Economics, Ashenfelter O.C., Card D. eds, North Holland, vol. 3., chap. 33, 2165-2214.

Sattinger M. [1993], "Assignments Models of the Distribution of Earnings", Journal of Economic Literature, vol. 31(2), 830-880.

Schmidt C.M., Zimmerman K.F. [1991], "Work Characteristics, Firm Size and Wages", The Review of Economics and Statistics, vol. 73, $\mathrm{n}^{\circ} 4,705-710$.

Soderbom M., Teal F., Wambugu A. [2005], "Unobserved Heterogeneity and the Relation Between Earnings and Firm Size: Evidence from Two Developing Countries", Economic letters, à paraître.

Stigler G.J. [1962], "Information in the Labor Market", Journal of Political Economy, vol. 70, n 5 - Part 2, 94-105.

Troske K.R. [1999], "Evidence on the Employer Size-Wage Premium from WorkerEstablishment Matched Data", The Review of Economic and Statistics, vol. 81, $\mathrm{n}^{\circ} 1$, $15-26$.

Weiss L. W. [1966], "Concentration and Labor Earnings", American Economic Review, vol. 56, 96-117.

White H. [1980], "A Heteroscedasticity Consistent Covariance Matrix Estimator and a Direct Test for Heteroscedasticity", Econometrica, vol. 48, 817-838.

Winter-Ebmer R., Zweimüller J. [1999], "Firm Size-Wage Differentials in Switzerland: Evidence from Job Changers", American Economic Review, vol. 89, $\mathrm{n}^{\circ}$ 2, 89-93. 
Annexe 1 - Travaux empiriques sur la relation salaire-taille

\begin{tabular}{|c|c|c|c|}
\hline Auteurs & Etablissement/Firme $^{\mathrm{a}}$ & Pays & $\begin{array}{c}\text { Type de données } \\
\text { et période }{ }^{\mathrm{a}}\end{array}$ \\
\hline Mellow W. (1982) & $(\mathrm{E}),(\mathrm{F})$ & USA & $(\mathrm{C}, 1979)$ \\
\hline Evans, Leighton (1988) & $(\mathrm{E}),(\mathrm{F})$ & USA & $(\mathrm{L}, 1976-81)$ \\
\hline Brown, Medoff $(1989)^{\mathrm{b}}$ & $(\mathrm{E}),(\mathrm{F})$ & USA & $(\mathrm{C}),(\mathrm{L})$ \\
\hline Gerlach, Schmidt (1990) & $(\mathrm{F})$ & RFA & $(\mathrm{L}, 1984-87)$ \\
\hline Idson, Feaster (1990) & $(\mathrm{F})$ & USA & $(\mathrm{C}, 1979)$ \\
\hline Schmidt, Zimmerman (1991) & $(\mathrm{F})$ & Autriche & $(\mathrm{C}, 1978)$ \\
\hline Main, Reilly (1993) & $(\mathrm{E})$ & Angleterre & $(\mathrm{C}, 1986)$ \\
\hline Davis, Haltiwanger (1996) & (E) & USA & $(\mathrm{C}, 1982)$ \\
\hline $\begin{array}{l}\text { Albaek, Arai, Asplund, Barth, } \\
\text { Strojer Madsen (1998) }\end{array}$ & $(\mathrm{E})$ & $\begin{array}{c}\text { Norvège, } \\
\text { Finlande, } \\
\text { Suède, } \\
\text { Danemark }\end{array}$ & $\begin{array}{l}(\mathrm{C}, 1989) \\
(\mathrm{C}, 1985) \\
(\mathrm{C}, 1991) \\
(\mathrm{C}, 1991)\end{array}$ \\
\hline Brunello, Colussi (1998) & $(\mathrm{F})$ & Italie & $(\mathrm{C}, 1993)$ \\
\hline $\begin{array}{l}\text { Abowd, Kramarz, Margolis } \\
\text { (1999) }\end{array}$ & $(\mathrm{E}),(\mathrm{F})$ & France & $(\mathrm{L}, 1976-87)$ \\
\hline Oi, Idson (1999b), & $(\mathrm{E}),(\mathrm{F})$ & USA & $(\mathrm{C}, 1983)$ \\
\hline $\begin{array}{l}\text { Winter-Ebmer R., Zweimüller } \\
\text { J. (1999) }\end{array}$ & $(\mathrm{F})$ & Suisse & $(\mathrm{L}, 1991-96)$ \\
\hline Troske K.R. (1999) & $(\mathrm{E})$ & USA & $(\mathrm{C}, 1990)$ \\
\hline Criscuolo C. (2000) & $(\mathrm{E})$ & Allemagne & $(\mathrm{C}, \mathrm{L}, 1984-95)$ \\
\hline Lluis S. (2003) & $(\mathrm{E}),(\mathrm{F})$ & Canada, USA & $(\mathrm{C}, 1998)$ \\
\hline
\end{tabular}

a- (E) : Etablissement ; (F) : Firme ; (C) : coupe ; (L) : Longitudinale

b- Brown et Medoff utilisent plusieurs bases de données portant sur diverses périodes. 


\section{Annexe 2 - Nomenclature des fonctions occupées}

$\mathrm{F} 1$ = production fabrication chantiers

$\mathrm{F} 2$ = installation entretien réglage réparation

F3 = nettoyage gardiennage travaux ménagers

$\mathrm{F} 4=$ manutention magasinage transports

F5 = secrétariat standard guichet saisie

F6 = gestion comptabilité fonction administrative

$\mathrm{F} 7=$ commerce vente technico-commercial

F8 = recherche études conseil

F9 = informatique télécommunications

F10 = directeur général ou adjoint direct cadre direction

F11 = enseignement santé information autre 
Tableau 1 : Statistiques descriptives ${ }^{a}$

\begin{tabular}{|c|c|c|c|c|c|c|c|c|c|c|c|c|}
\hline & \multicolumn{2}{|c|}{1 à $2 \mathrm{sal}$} & \multicolumn{2}{|c|}{3 à 9 sal } & \multicolumn{2}{|c|}{10 à 49 sal } & \multicolumn{2}{|c|}{50 à 199 sal } & \multicolumn{2}{|c|}{200 à 499 sal } & \multicolumn{2}{|c|}{500 sal et +} \\
\hline & moy & (e.t) & moy & (e.t) & moy & (e.t) & moy & (e.t) & moy & (e.t) & moy & (e.t) \\
\hline Ln(SALAIRE) & 6,850 & $(0,261)$ & 6,903 & $(0,255)$ & 6,959 & $(0,290)$ & 7,039 & $(0,318)$ & 7,122 & $(0,337)$ & 7,235 & $(0,346)$ \\
\hline PLURI & 0,212 & $(0,409)$ & 0,320 & $(0,467)$ & 0,488 & $(0,500)$ & 0,627 & $(0,484)$ & 0,723 & $(0,448)$ & 0,833 & $(0,373)$ \\
\hline SEXE & 0,611 & $(0,488)$ & 0,579 & $(0,494)$ & 0,594 & $(0,491)$ & 0,576 & $(0,494)$ & 0,571 & $(0,495)$ & 0,638 & $(0,481)$ \\
\hline CSP1 & 0,275 & $(0,447)$ & 0,210 & $(0,407)$ & 0,181 & $(0,385)$ & 0,162 & $(0,368)$ & 0,150 & $(0,358)$ & 0,137 & $(0,343)$ \\
\hline CSP2 & 0,189 & $(0,391)$ & 0,255 & $(0,436)$ & 0,276 & $(0,447)$ & 0,318 & $(0,466)$ & 0,348 & $(0,476)$ & 0,421 & $(0,494)$ \\
\hline CSP3 & 0,536 & $(0,499)$ & 0,535 & $(0,499)$ & 0,543 & $(0,498)$ & 0,521 & $(0,500)$ & 0,502 & $(0,500)$ & 0,443 & $(0,497)$ \\
\hline EDUC & 14,801 & $(2,585)$ & 15,146 & $(2,807)$ & 15,431 & $1(2,797)$ & 15,900 & $(2,853)$ & 16,228 & $8(2,749)$ & 16,544 & $4(2,841)$ \\
\hline DIPLOME & 0,677 & $(0,468)$ & 0,699 & $(0,459)$ & 0,701 & $(0,458)$ & 0,749 & $(0,434)$ & 0,783 & $(0,412)$ & 0,792 & $(0,406)$ \\
\hline EXPE & 10,863 & $(10,684)$ & ) 11,299 & $(10,730)$ & 11,108 & $8(10,426)$ & 10,974 & $4(10,395)$ & 10,158 & $8(10,075)$ & 9,275 & $(10,094)$ \\
\hline INDEP- $^{-}$ & 0,443 & $(0,497)$ & 0,512 & $(0,500)$ & 0,602 & $(0,489)$ & 0,662 & $(0,473)$ & 0,676 & $(0,468)$ & 0,689 & $(0,463)$ \\
\hline INDEP $^{+}$ & 0,225 & $(0,418)$ & 0,230 & $(0,421)$ & 0,224 & $(0,417)$ & 0,200 & $(0,400)$ & 0,211 & $(0,408)$ & 0,209 & $(0,406)$ \\
\hline $\mathrm{INDEP}^{++}$ & 0,310 & $(0,462)$ & 0,241 & $(0,428)$ & 0,167 & $(0,373)$ & 0,134 & $(0,340)$ & 0,109 & $(0,312)$ & 0,099 & $(0,299)$ \\
\hline $\mathrm{RESP}^{-}$ & 0,871 & $(0,336)$ & 0,780 & $(0,414)$ & 0,770 & $(0,421)$ & 0,741 & $(0,438)$ & 0,727 & $(0,446)$ & 0,740 & $(0,439)$ \\
\hline $\mathrm{RESP}^{+}$ & 0,127 & $(0,333)$ & 0,204 & $(0,403)$ & 0,183 & $(0,387)$ & 0,193 & $(0,395)$ & 0,187 & $(0,390)$ & 0,189 & $(0,392)$ \\
\hline $\mathrm{RESP}^{++}$ & 0,003 & $(0,053)$ & 0,016 & $(0,125)$ & 0,047 & $(0,212)$ & 0,066 & $(0,248)$ & 0,086 & $(0,281)$ & 0,071 & $(0,256)$ \\
\hline PAIE & 0,0383 & $(0,192)$ & 0,044 & $(0,205)$ & 0,048 & $(0,190)$ & 0,034 & $(0,182)$ & 0,048 & $(0,213)$ & 0,058 & $(0,234)$ \\
\hline URBAIN & 0,732 & $(0,443)$ & 0,813 & $(0,390)$ & 0,840 & $(0,367)$ & 0,839 & $(0,367)$ & 0,864 & $(0,342)$ & 0,890 & $(0,313)$ \\
\hline NEMP & 2,228 & $(1,349)$ & 2,254 & $(1,311)$ & 2,272 & $(1,306)$ & 2,218 & $(1,261)$ & 2,102 & $(1,155)$ & 1,973 & $(1,087)$ \\
\hline $\mathrm{N}=26694$ & 1399 & & 5250 & & 7927 & & 5602 & & 3045 & & 3471 & \\
\hline
\end{tabular}

a- Les variables muettes relatives aux filières de formation suivies par les individus, aux fonctions occupées, aux secteurs d'activité et aux régions de localisation de l'emploi ne figurent pas dans ce tableau. 
Tableau 2 : Equations de gains avec prise en compte de la taille des établissements

\begin{tabular}{|c|c|c|c|c|c|c|c|c|}
\hline & $(1)^{\mathrm{b}}$ & $(2)^{b}$ & $(3)^{\mathrm{b}}$ & $(4)^{b}$ & $(5)^{\mathrm{b}}$ & $(6)^{b}$ & $(7)^{b}$ & $(8)^{b}$ \\
\hline \multirow[t]{2}{*}{ 3-9 SAL. } & 0,0443 & 0,0409 & 0,0364 & 0,0300 & 0,0246 & & 0,0178 & \\
\hline & $(5,57)$ & $(5,52)$ & $(4,96)$ & $(4,089)$ & $(3,37)$ & & $(2,35)$ & \\
\hline \multirow[t]{2}{*}{ 10-49 SAL. } & 0,0872 & 0,0681 & 0,0634 & 0,0550 & 0,0451 & & 0,0394 & \\
\hline & $(11,13)$ & $(9,31)$ & $(8,73)$ & $(7,52)$ & $(6,20)$ & & $(5,15)$ & \\
\hline \multirow[t]{2}{*}{ 50-199 SAL. } & 0,1499 & 0,1064 & 0,0985 & 0,0877 & 0,0721 & & 0,0665 & \\
\hline & $(18,24)$ & $(13,88)$ & $(12,95)$ & $(11,39)$ & $(9,457)$ & & $(7,75)$ & \\
\hline \multirow[t]{2}{*}{ 200-499 SAL. } & 0,2208 & 0,1500 & 0,1416 & 0,1288 & 0,1073 & & 0,0899 & \\
\hline & $(24,16)$ & $(17,86)$ & $(17,11)$ & $(15,39)$ & $(12,92)$ & & $(8,47)$ & \\
\hline \multirow[t]{2}{*}{500 SAL. ET PLUS } & 0,3113 & 0,2138 & 0,2039 & 0,1855 & 0,1543 & & 0,1315 & \\
\hline & $(34,40)$ & $(25,73)$ & $(24,80)$ & $(22,11)$ & $(18,48)$ & & $(10,79)$ & \\
\hline \multirow[t]{2}{*}{$\operatorname{Ln}(\text { EFFECTIFS })^{\text {a }}$} & & & & & & 0,0221 & & 0,0206 \\
\hline & & & & & & $(25,32)$ & & $(22,52)$ \\
\hline \multirow[t]{2}{*}{ PLURI } & & & & & & & & 0,0177 \\
\hline & & & & & & & & $(5,59)$ \\
\hline \multirow[t]{2}{*}{ (3-9 SAL)x PLURI } & & & & & & & 0,0221 & \\
\hline & & & & & & & $(3,37)$ & \\
\hline \multirow[t]{2}{*}{ (10-49 SAL)xPLURI } & & & & & & & 0,0126 & \\
\hline & & & & & & & $(2,39)$ & \\
\hline \multirow[t]{2}{*}{ (50-199 SAL)xPLURI } & & & & & & & 0,0095 & \\
\hline & & & & & & & $(1,50)$ & \\
\hline \multirow[t]{2}{*}{ (200-499 SAL)x PLURI } & & & & & & & 0,0251 & \\
\hline & & & & & & & $(2,67)$ & \\
\hline \multirow[t]{2}{*}{ (500 SAL ET + )x PLURI } & & & & & & & 0,0285 & \\
\hline & & & & & & & $(2,65)$ & \\
\hline $\mathrm{R}^{2}$ & 0,322 & 0,476 & 0,503 & 0,510 & 0,537 & 0,536 & 0,538 & 0,537 \\
\hline $\mathrm{N}$ & 24338 & 24338 & 24338 & 24338 & 24338 & 24338 & 24338 & 24338 \\
\hline
\end{tabular}

a- Logarithme naturel de la taille estimée à partir des centres de classes.

b- $t$ statistiques entre parenthèses. Calculées à partir des écarts-type corrigés de l'hétéroscédasticité selon la procédure de White [1980].

Note : Les régressions incluent, outre les variables de taille :

Col. 1 : constante, variables de contrôle pour les caractéristiques individuelles et de capital humain : sexe, educ, expe, $(\text { expe })^{2}$, indep + , indep ++ .

Col. 2: constante, variables de contrôle pour les caractéristiques individuelles et de capital humain : sexe, filières*spécialités*diplômes (42 variables muettes), expe, (expe) ${ }^{2}$, indep+, indep++.

Col. 3 : idem (2) et variables de contrôle pour l'effort du salarié (resp+, resp++) et les fonctions occupées (10 variables muettes).

Col. 4 : idem (3) et variables de contrôle pour secteurs d'activité (15 variables muettes).

Col. 5 : idem (4) et variables de contrôle pour dimension spatiale : régions de localisation de l'emploi (22 variables muettes), urbain.

Col. 6,7,8: mêmes variables de contrôle que (5). 
Tableau 3 : Estimation en différences premières des effets de la taille sur l'écart entre le dernier et le premier emploi occupés

\begin{tabular}{|c|c|c|c|c|c|}
\hline & $(1)^{d}$ & $(2)^{\mathrm{d}}$ & $(3)^{d}$ & $(4)^{d}$ & $(5)^{d}$ \\
\hline \multirow{2}{*}{ 3-9 SAL } & 0,0118 & & 0,0042 & & 0,0093 \\
\hline & $(0,91)$ & & $(0,37)$ & & $(0,81)$ \\
\hline \multirow{2}{*}{$10-49 \mathrm{SAL}$} & 0,0380 & & 0,0282 & & 0,0355 \\
\hline & $(3,00)$ & & $(2,49)$ & & $(3,12)$ \\
\hline \multirow{2}{*}{ 50-199 SAL } & 0,0486 & & 0,0317 & & 0,0397 \\
\hline & $(3,68)$ & & $(2,63)$ & & $(3,29)$ \\
\hline \multirow{2}{*}{ 200-499 SAL } & 0,0902 & & 0,0654 & & 0,0795 \\
\hline & $(6,27)$ & & $(4,95)$ & & $(6,03)$ \\
\hline \multirow{2}{*}{$500 \mathrm{SAL} \mathrm{ET}+$} & 0,1338 & & 0,0923 & & 0,1084 \\
\hline & $(9,30)$ & & $(6,93)$ & & $(8,20)$ \\
\hline \multirow{2}{*}{ Ln(EFFECTIFS) } & & 0,0195 & & 0,0139 & \\
\hline & & $(12,83)$ & & $(9,53)$ & \\
\hline \multirow{2}{*}{ MOBILITÉ PROF ${ }^{\mathrm{a}}$} & & & & & $-0,0072$ \\
\hline & & & & & $(-1,13)$ \\
\hline \multirow{2}{*}{ MOBILITÉ SECT b } & & & & & 0,0059 \\
\hline & & & & & $(0,96)$ \\
\hline \multirow{2}{*}{ MOBILITÉ GÉO ${ }^{c}$} & & & & & 0,0334 \\
\hline & & & & & $(4,97)$ \\
\hline $\mathrm{R}^{2}$ & 0,576 & 0,576 & 0,066 & 0,064 & 0,049 \\
\hline $\mathrm{N}$ & 7742 & 7742 & 7390 & 7390 & 5280 \\
\hline
\end{tabular}

a- Indicatrice. Vaut 1 si l'individu n'occupe plus la même fonction, 0 sinon.

b- Indicatrice. Vaut 1 si l'individu ne travaille plus dans le même secteur, 0 sinon.

c- Indicatrice. Vaut 1 si l'individu ne travaille plus dans la même région, 0 sinon.

d- $t$ statistiques entre parenthèses.

Note : Les régressions incluent :

Col. (1) et (2) : Variable dépendante : logarithme du salaire. Variables de contrôle : sexe, filières*spécialités*diplômes (42), expe, (expe) $)^{2}$ indep + , indep ++ , fonctions (10), resp + , resp ++ , secteurs (15), régions (22), urbain.

Col. (3) et (4) : Variable dépendante : écart dans le logarithme du salaire. Variables de contrôle : écarts dans les variables sexe, filières* ${ }^{*}$ spécialités*diplômes (42), expe, (expe) ${ }^{2}$, indep + , indep ++ , fonctions (10), resp + , resp ++ , secteurs (15), régions $(22)$, urbain. Les écarts sont nuls pour les variables sexe, filières de formation, indep + et indep ++ .

Col. (5) : Variable dépendante : écart dans le logarithme du salaire. Variables de contrôle : écarts dans les variables sexe, filières*spécialités*diplômes, expe, $(\text { expe })^{2}$, indep + , indep ++ , resp + , resp ++ , urbain. Les écarts sont nuls pour les variables sexe, filières de formation, indep+ et indep ++ . 
Tableau 4 : Equation de sélection (probit ordonné) ${ }^{\mathrm{a}}$

\begin{tabular}{lc}
\hline & Coeff. $^{\text {b }}$ \\
\hline CONSTANTE & $-0,1914$ \\
EDUC & $(-3,81)$ \\
& 0,0595 \\
NEMP & $(21,39)$ \\
& $-0,0342$ \\
COUPLE & $(-5,76)$ \\
& $-0,0052$ \\
CSP2 & $(-0,34)$ \\
& 0,2763 \\
CSP3 & $(12,67)$ \\
& 0,1376 \\
INDEP & $(8,82)$ \\
& $-0,1418$ \\
INDEP & $(-7,82)$ \\
& $-0,4329$ \\
Mu 1 & $(-21,48)$ \\
& 0,8165 \\
Mu 2 & $(91,93)$ \\
Khi-deux & 1,4138 \\
N & $(129,05)$ \\
\hline a- vaiable & 1408,7 \\
& 22280 \\
\hline
\end{tabular}

a- variable dépendante : taille de l'établissement (quatre modalités)

b- $t$ statistiques entre parenthèses 
Tableau 5 : Equations de gains par classe de taille d'établissement

\begin{tabular}{|c|c|c|c|c|c|c|}
\hline & \multicolumn{2}{|c|}{ Sans correction pour sélection } & \multicolumn{4}{|c|}{ Avec correction pour sélection } \\
\hline & (1) & (2) & (3) & (4) & (5) & (6) \\
\hline & 1-9 sal. ${ }^{\mathrm{a}}$ & $\begin{array}{l}200 \text { sal. et } \\
\text { plus }^{\mathrm{a}}\end{array}$ & $1-9$ sal. $^{a}$ & $\begin{array}{c}200 \text { sal. et } \\
\text { plus }^{\mathrm{a}}\end{array}$ & 1-9 sal. ${ }^{\mathrm{a}}$ & $\begin{array}{c}200 \text { sal. et } \\
\text { plus }^{\mathrm{a}}\end{array}$ \\
\hline \multirow[t]{2}{*}{ CONSTANTE } & 7,2441 & 7,3656 & 6,9816 & 7,5479 & 6,4014 & 7,0476 \\
\hline & $(173,45)$ & $(314,76)$ & $(124,13)$ & $(136,72)$ & $(158,35)$ & $(74,18)$ \\
\hline \multirow[t]{2}{*}{ SEXE } & 0,0864 & 0,0792 & 0,0842 & 0,0756 & 0,0759 & 0,0595 \\
\hline & $(10,57)$ & $(10,62)$ & $(10,62)$ & $(7,49)$ & $(9,74)$ & $(7,55)$ \\
\hline \multirow[t]{2}{*}{ EDUC } & & & & & 0,0175 & 0,0210 \\
\hline & & & & & $(7,23)$ & $(8,01)$ \\
\hline \multirow[t]{2}{*}{ DIPLÔME } & & & & & 0,0444 & 0,1016 \\
\hline & & & & & $(6,65)$ & $(11,56)$ \\
\hline \multirow[t]{2}{*}{$(\mathrm{EXPE}) \times 10$} & 0,0142 & 0,0151 & 0,0295 & 0,0282 & 0,0294 & 0,0099 \\
\hline & $(1,63)$ & $(1,52)$ & $(3,20$ & $(2,07)$ & $(3,04)$ & $(0,88)$ \\
\hline \multirow[t]{2}{*}{$(\mathrm{EXPE})^{2} \times 100$} & 0,0063 & 0,0111 & 0,0022 & 0,0075 & 0,0018 & 0,0108 \\
\hline & $(2,12)$ & $(3,04)$ & $(0,70)$ & $(1,53)$ & $(0,55)$ & $(2,67)$ \\
\hline \multirow[t]{2}{*}{ INDEP $^{+}$} & 0,0192 & 0,0187 & 0,0411 & 0,0374 & 0.0453 & 0,0774 \\
\hline & $(2,79)$ & $(2,37$ & $(5,24)$ & $(3,29)$ & $(5.14)$ & $(8,00)$ \\
\hline \multirow[t]{2}{*}{$\mathrm{INDEP}^{++}$} & 0,0449 & 0,0241 & 0,1042 & 0,0810 & 0.1060 & 0,1444 \\
\hline & $(6,17)$ & $(2,29$ & $(9,23)$ & $(4,04)$ & $(6.83)$ & $(7,40)$ \\
\hline \multirow[t]{2}{*}{$\mathrm{RESP}^{+}$} & 0,0615 & 0,0559 & 0,0571 & 0,0561 & 0,0690 & 0,0781 \\
\hline & $(8,29)$ & $(7,31)$ & $(7,59)$ & $(5,232)$ & $(8,82)$ & $(8,86)$ \\
\hline \multirow[t]{2}{*}{$\mathrm{RESP}^{++}$} & 0,0967 & 0,0901 & 0,0939 & 0,0771 & 0,1189 & 0,1118 \\
\hline & $(3,67)$ & $(7,32$ & $(3,82)$ & $(4,87)$ & $(4,66)$ & $(8,62)$ \\
\hline \multirow[t]{2}{*}{ PluRI } & 0,0252 & 0,0305 & 0,0268 & 0,0305 & 0,0290 & 0,0389 \\
\hline & $(3,84)$ & $(4,10)$ & $(3,87)$ & $(2,91)$ & $(4,03)$ & $(4,51)$ \\
\hline \multirow[t]{2}{*}{$\lambda$} & - & - & $-0,1738$ & $-0,1674$ & $-0,1657$ & $-0,3405$ \\
\hline & & & $(-6,53)$ & $(-4,13)$ & $(-3,96)$ & $(-7,35)$ \\
\hline $\mathrm{R}^{2}$ & 0,345 & 0,574 & 0,350 & 0,578 & 0,296 & 0,483 \\
\hline $\mathrm{N}$ & 5928 & 6060 & 5430 & 5617 & 5366 & 5553 \\
\hline
\end{tabular}

a- $t$ statistiques entre parenthèses. Calculées à partir des écarts-type corrigés de l'hétéroscédasticité.

Note : Les régressions incluent également :

col (1) à (4) : 42 variables muettes pour formation et diplôme, 10 variables muettes pour fonctions occupées, 15 pour secteurs d'activité, 22 pour régions de localisation de l'emploi, 1 pour localisation urbaine ou non de l'emploi.

col (5) et (6) : idem précédent sauf variables muettes pour formation et diplôme. 